\title{
Obesity resistant mechanisms in the Lean polygenic mouse model as indicated by liver transcriptome and expression of selected genes in skeletal muscle
}

Matjaž Simončič ${ }^{1}$, Tadeja Režen ${ }^{2}$, Peter Juvan², Damjana Rozman², Gregor Fazarinc ${ }^{3}$, Catherine Fievet ${ }^{4,5,6,7}$, Bart Staels ${ }^{4,5,6,7}$, Simon Horvat ${ }^{1,8^{*}}$

\begin{abstract}
Background: Divergently selected Lean and Fat mouse lines represent unique models for a polygenic form of resistance and susceptibility to obesity development. Previous research on these lines focused mainly on obesitysusceptible factors in the Fat line. This study aimed to examine the molecular basis of obesity-resistant mechanisms in the Lean line by analyzing various fat depots and organs, the liver transcriptome of selected metabolic pathways, plasma and lipid homeostasis and expression of selected skeletal muscle genes.

Results: Expression profiling using our custom Steroltalk v2 microarray demonstrated that Lean mice exhibit a higher hepatic expression of cholesterol biosynthesis genes compared to the Fat line, although this was not reflected in elevation of total plasma or liver cholesterol. However, FPLC analysis showed that protective HDL cholesterol was elevated in Lean mice. A significant difference between the strains was also found in bile acid metabolism. Lean mice had a higher expression of Cyp8b1, a regulatory enzyme of bile acid synthesis, and the Abcb11 bile acid transporter gene responsible for export of acids to the bile. Additionally, a higher content of blood circulating bile acids was observed in Lean mice. Elevated HDL and upregulation of some bile acids synthesis and transport genes suggests enhanced reverse cholesterol transport in the Lean line - the flux of cholesterol out of the body is higher which is compensated by upregulation of endogenous cholesterol biosynthesis. Increased skeletal muscle $1 / 6$ and Dio2 mRNA levels as well as increased activity of muscle succinic acid dehydrogenase (SDH) in the Lean mice demonstrates for the first time that changes in muscle energy metabolism play important role in the Lean line phenotype determination and corroborate our previous findings of increased physical activity and thermogenesis in this line. Finally, differential expression of Abcb11 and Dio2 identifies novel strong positional candidate genes as they map within the quantitative trait loci (QTL) regions detected previously in crosses between the Lean and Fat mice.
\end{abstract}

Conclusion: We identified novel candidate molecular targets and metabolic changes which can at least in part explain resistance to obesity development in the Lean line. The major difference between the Lean and Fat mice was in increased liver cholesterol biosynthesis gene mRNA expression, bile acid metabolism and changes in selected muscle genes' expression in the Lean line. The liver Abcb11 and muscle Dio2 were identified as novel positional candidate genes to explain part of the phenotypic difference between the Lean and Fat lines.

\footnotetext{
* Correspondence: simon.horvat@bf.uni-lj.si

'University of Ljubljana, Biotechnical Faculty, Department of Animal Science, Groblje 3, 1230 Domžale, Slovenia

Full list of author information is available at the end of the article
} 


\section{Background}

The 20th century is marked by an explosive increase in obesity and metabolic syndrome commencing in populations of the Western world in the 1960's and is becoming a significant burden also in developing nations [1]. Metabolic syndrome encompasses a cluster of deleterious metabolic events characterized by obesity, hyperglycemia, insulin resistance, hypercholesterolemia, hypertriglyceridemia and hypertension. Environmental determinants influencing the development of the metabolic syndrome include low-cost highly palatable foods and the increasing mechanization of transport and manual tasks following the industrial revolution. While the intake of calories gradually declined over the past decades, physical inactivity and its interaction with genetic predisposition to obesity seem to be an increasingly prevalent determinant of metabolic syndrome development $[2,3]$.

Previous research in the field of metabolic syndrome and obesity primarily studied monogenic knockout rodent models [4]. Although murine models with singlegene mutations are useful to study the effects of a few genes with major effect on human obesity, monogenic defects account for only a minority of patients with morbid obesity. In contrast, epidemic proportions of the metabolic syndrome and obesity are ascribed to the polygenic effects, where multiple genes interact with many environmental factors over time $[5,6]$. Thus, single gene animal models can not explain a large portion of genetic variation that exists in the polygenic form making polygenic obesity mouse models $[7,8]$, such as the lines used herein, a more suitable genetic resource.

Accordingly, we employed Lean and Fat mouse lines developed from the same base population by long-term (over 60 generations) divergent selection for low or high body fat \% [9]. These Fat and Lean mouse lines may represent a unique model of the prevalent polygenic form of human obesity and leanness. Because previous research focused mainly on investigating obesity-susceptibility in the Fat line (e.g., [10-12]), we here aimed to elucidate the molecular basis of obesity-resistance in the Lean line.

We performed a thorough phenotypic characterization of the Lean and Fat lines. In addition, a geneexpression analysis was conducted on the liver tissue using the Steroltalk v2 microarray that was previously used to study hepatic lipid homeostasis [13-15]. This custom microarray is designed for detailed studies of genes from the cholesterol homeostasis and drug metabolism networks, including bile acid metabolism, and several genes from the fatty acid and glucose pathways as well as related transporters for the aforementioned processes.
The results of our study combined with previous findings help to identify, at least in part, a molecular basis for the contrasting phenotype between the Lean and Fat mice and substantiate a hypothesis that differences in metabolic interactions between skeletal muscle and liver tissues play an important part in the phenotype divergence between these lines. The Lean mouse line exhibits characteristics that uncover some of the factors involved in genetic predisposition for obesity-resistance that may also be relevant for obesity and metabolic syndrome basic and applied research in humans.

\section{Methods}

\section{Animals and maintenance}

Inbred lean (Lean) and fat (Fat) mouse lines used here were developed by long-term (over 60 generations) divergent selection for low or high body fat $\%[9,16]$. Our previous genetic studies demonstrated that these lines differ in large part due to cumulative action of multiple quantitative trait loci (QTLs) of small to medium sized effects, confirming that these lines may present a valuable model of the polygenic form of leanness/ obesity [17-19].

Animals were weaned at 3 weeks of age and one pair of males (usually brothers) was housed per cage. Cages were randomly distributed on the rack in order to minimize localized environmental effects and mice were

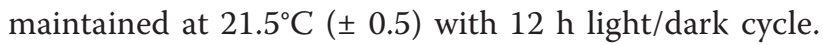
Mice were fed maintenance chow diet (Altromin 1324, Lage, Germany), containing: crude oil, $4 \%$; crude protein, $19 \%$; crude fibre, $6 \%$; ash, $7 \%$; moisture, $13.5 \%$; metabolizable energy, $11.9 \mathrm{MJ} / \mathrm{kg}$ where $24 \%, 11 \%$ and $65 \%$ of calories from protein, fat and carbohydrates, respectively. Body weights were recorded at 3, 6, 10 and 14 weeks of age. All procedures on animals were performed according to local ethical and regulatory guidelines, which are all in compliance with the EU regulations regarding research on experimental animals.

\section{Phenotype measurements and analyses}

At 14 weeks of age ( \pm 3 days) a total of 43 Lean and 42 Fat males were sacrificed after four hour fast by decapitation. Samples of blood, liver, entire left epididymal, left abdominal (perirenal and retroperitoneal), left femoral fat, mesenterial fat, heart and striated muscle from regio cruris (m.gastrocnemius) and thigh muscle (m. quadriceps) were weighed, promptly frozen in liquid nitrogen and stored at $-80^{\circ} \mathrm{C}$.

Blood was collected in Li-heparin tubes (Sarstedt, Germany) and centrifuged within $3 \mathrm{~h}$ of collection at 3000 $\mathrm{rpm} / \mathrm{min}$, for $10 \mathrm{~min}$ at $+4^{\circ} \mathrm{C}$. Plasma samples of 10 Lean and 10 Fat mice were individually analyzed for total plasma cholesterol, HDL-cholesterol, plasma 
triglyceride and glucose concentrations. Plasma total cholesterol and triglyceride concentrations were determined by enzymatic assays adapted to microtiter plates using commercially available reagents (BioMerieux, Lyon, France). Plasma HDL-cholesterol levels were measured after precipitation of apolipoprotein (Apo) B-containing lipoproteins with phosphotungstic acid/Mg (Roche Diagnostics GmbH, Mannheim, Germany). NonHDL cholesterol was obtained by subtraction of HDLcholesterol values from total plasma cholesterol.

Plasma lipoproteins were separated by gel filtration chromatography using a Superose 6 HR 10/30 column (Pharmacia, Sweden). The gel was allowed to equilibrate with $10 \mathrm{mM}$ phosphate-buffered saline (PBS) containing $0.01 \%$ (wt/vol) EDTA and $0.01 \%$ (wt/vol) sodium azide. Plasma was eluted with the buffer at room temperature at a flow rate of $0.2 \mathrm{ml}$ per minute. The effluents were collected in $0.22 \mathrm{ml}$ fractions. Cholesterol and triglyceride concentrations were determined in the eluted fractions as described above.

Cholesterol distribution among lipoproteins was obtained by separation of the major lipoprotein classes (VLDL, IDL+LDL, and HDL) by fast protein liquid chromatography (FPLC) carried out on $200 \mu \mathrm{l}$ of plasma sample for Fat and Lean line, according to procedure previously detailed [12]. Cholesterol concentrations were determined in the eluted fractions.

Analysis of hepatic lipids in individual samples of 10 Lean and 10 Fat mice was carried out using frozen liver tissue $(50 \mathrm{mg}$ ) which was homogenized in SET buffer ( $1 \mathrm{~mL}$; sucrose $250 \mathrm{mM}$, EDTA $2 \mathrm{mM}$ and Tris $10 \mathrm{mM}$ ), followed by two freeze-thaw cycles and three passages through a 27-gauge syringe needle and a final freezethaw cycle to ensure complete cell lysis. Protein content was determined with the BCA method and triglyceride and cholesterol was measured as described above.

Differences between the lines in all measured phenotypic parameters were tested by an unpaired Student $t$-test on untransformed data as the data exhibited normal distribution.

\section{SDH activity of striated muscles}

Oxidative capacity of the muscle fibres (mid-portion of m. gastrocnemius) was determined by the mitochondrial succinate dehydrogenase (SDH) assay as described previously [20]. Briefly, $10 \mu \mathrm{m}$ thick cryostat sections were incubated for 45 minutes at $37^{\circ} \mathrm{C}$ in a moisture chamber with the medium composed of equal volumes of $0.2 \mathrm{M}$ phosphate buffer saline ( $\mathrm{pH}$ 7.6), $0.2 \mathrm{M}$ disodium succinate and aqueous solution of nitro-BT $(1 \mathrm{mg} / \mathrm{ml})$. After incubation the slides were washed in tap water, dehydrated and mounted with Synthetic Mountant (Shandon, USA). SDH has an important role in metabolism of tricarbonic acids by the Krebs cycles. In the histochemical reaction the enzyme takes over the hydrogen from disodium succinate and transfers it to the tetrasolium salt (NBT), which turns visible in the form of blue formazan granules.

To analyze the SDH activity in muscle fibres a Nikon Microphot FXA microscope (Nikon Instruments Europe BV, Badhoevendorp, The Netherlands) and the Lucia-G analyzing software (Laboratory Imaging, Prague, Czech Republic) were used. In total 12 of each Lean and Fat line mice were analyzed. 150 muscle fibres of each mouse were measured by densitometry. Higher \% densitometry indicated higher transparency resulting from lower SDH-enzyme activity.

\section{RNA isolation}

10 liver samples were individually homogenized for $10 \mathrm{~s}$ using an Ultra-Turrax T8 homogenizer (IKA Labortechnik, Germany) and RNA extracted in »batches « (a pair of Fat and Lean line samples simultaneously) using TRIzol reagent (TRIzol ${ }^{\circledR}$ reagent, Invitrogen, Life technologies). RNA quality was evaluated by ND-1000 spectrophotometer (Nanodrop Technologies, Wilmington, USA) and Agilent 2100 bioanalyzer. According to the given RINs (RNA integrity number) a pairwise comparison between Fat and Lean line RNA samples was made in order to couple samples with least quality divergence. RIN values of all samples ranged from 7.5-8.5 suggesting low variability and high overall quality. Maximal tolerated RNA quality divergence for a pair of Lean and Fat-line samples co-hybridized on the same microarray was \pm 0.5 RIN.

\section{Microarray experiment}

The Steroltalk v2 cDNA microarray used here was a next generation of the previously described Sterolgene v0 array [14] with additional cDNA probes detecting a total of 278 mouse genes from selected metabolic pathways relevant to obesity research. The design and preparation of these additional probes was the same as described [14]. The Steroltalk v2 cDNA microarray was focused on targeted functional groups such as enzymes from cholesterol biosynthesis (18/21 probes; coverage $85.7 \%)$, nuclear receptor families ( $45 / 49$ probes; coverage 93.75\%; http://www.nursa.org/) and members of the cytochrome P450 (38/103 probes, coverage 36.9\%) [14]. Additionally, key enzymes of glucose and fatty acid metabolism; transporters of xenobiotics, cholesterol and bile acids; and proteins involved in cholesterol plasma transport, circadian rhythm, and selected cell signalling pathways involved in the regulation of cholesterol homeostasis were included. A full list of genes contained on the custom Steroltalk v2 array along with associated functional groups is provided in Additional file 1. 
Individual total RNA samples from 10 Lean and 10 Fatline animals were labelled using $\mathrm{Cy} 3$ and $\mathrm{Cy} 5$ dye. To $20 \mu \mathrm{g}$ of Lean and Fat line total RNA sample, spike of control RNAs was added: 250 pg of Firefly Luciferase mRNA (Promega, Madison, WI, USA) and $0.5 \mu \mathrm{L}$ of either test or reference spike mix from Lucidea Universal Scorecard kit (Amersham Biosciences, GE Healthcare UK limited, Little Chalfont, UK). mRNA was reverse transcribed to aminoallyl cDNA using $2.5 \mu \mathrm{g}$ of Oligo dT (Invitrogen, Carlsbad, CA, USA), 400 U of SuperScript ${ }^{\mathrm{TM}}$ III Reverse Transcriptase (Invitrogen, Carlsbad, CA, USA) and $1 \mu \mathrm{L}$ of $10 \mathrm{mM}$ amino-allyl dUTP (Sigma, St Louis, MI, USA) according to the manufacturer's protocol. Reactions were stopped after 2 hours by addition of $10 \mu \mathrm{L}$ of $0.5 \mathrm{M}$ EDTA and of $1 \mathrm{M} \mathrm{NaOH}$ and were further incubated at $65^{\circ} \mathrm{C}$ for $15 \mathrm{~min} .10 \mu \mathrm{L}$ of $1 \mathrm{M} \mathrm{HCl}$ was added and cDNA was purified using MinElute PCR Purification Kit (Qiagen $\mathrm{GmBH}$, Hilden, D) according to the manufacturer's protocol with exception of using a phosphate buffer $\left(5 \mathrm{mM} \mathrm{KPO}_{4} \mathrm{pH}\right.$ 8.5 in $80 \%$ ethanol) for the washing step and MilliQ water for elution. Purified amino-allyl cDNA was dried and resuspended in $4.5 \mu \mathrm{L}$ of $0.2 \mathrm{M} \mathrm{Na}_{2} \mathrm{CO}_{3}(\mathrm{pH} 9.0)$ and $4.5 \mu \mathrm{L}$ of cyanin-3 or cyanin-5 dye in DMSO (Amersham Biosciences, GE Healthcare UK limited, Little Chalfont, UK). The labelling reaction was incubated at room temperature for two hours, precipitated by addition of $35 \mu \mathrm{L}$ of $0.1 \mathrm{M} \mathrm{Na}$ acetate ( $\mathrm{pH}$ 5.2) and purified reaction using a MinElute PCR Purification Kit (Qiagen GmBH, Hilden, D) were added. Labelled cDNA was eluted in water and $1 \mu \mathrm{L}$ was used for evaluation on ND-1000 spectrophotometer (Nanodrop Technologies, Wilmington, Delaware, USA). 10 Lean and Fat line cDNA samples, respectively, were cohybridized in pairs and dye swaps were performed. Using LifterSlip cover glasses (Erie Scientific Company, Portsmouth, NH, USA) samples were hybridized for $16 \mathrm{~h}$ at $65^{\circ} \mathrm{C}$ using buffers $3 \times \mathrm{SSC}$ and $0.2 \%$ SDS in humidified hybridization chambers (HybChambers, GeneMachines, San Carlos, CA, USA). The slides were washed and scanned as described [14].
Images were analyzed using Array-Pro Analyzer 4.5 (Media Cybernetics, Bethesda, MD, USA). Three arrays did not pass quality control parameters (mainly too high signal to noise ratios) and hence 17 arrays were included in downstream statistical analyses. Data were normalized using OWNormalize, a widget for explorative normalization of focused microarrays, implemented within Orange software [21]. Data were first filtered to exclude spots of low quality and then normalized using LOWESS fit to "spike in" control RNAs (Firefly luciferase and Lucidea Universal Scorecard) according to their average intensity. The raw and normalized gene expression data of 17 arrays together with experimental information are deposited in Gene Expression Omnibus database (http://www.ncbi.nlm.nih.gov/geo/, accession number GSE24967) in compliance with MIAME standards [22]. Differential expression was assessed using Student's t-test at probability of type I error $\alpha=0.05$. Expression of genes was considered on individual basis and therefore no multiple testing correction has been applied.

\section{Quantitative reverse transcription polymerase chain reaction (qRT-PCR)}

A random selection of differentially expressed genes from the microarray experiment and some genes not represented on the array (for assaying in muscle tissue) were assayed by the qRT-PCR to validate array results (Table 1 Table 2, and Figure 1). The same liver RNAs were used as originally in the array experiments. In analyses of muscle genes, 8 and 10 RNAs from the Lean and Fat were used, respectively - again, using the same animals as used in the array experiment. Briefly, $1 \mu \mathrm{g}$ of total RNA was DNAse I (Sigma, St Louis, MI, USA) treated, reverse-transcribed (SuperScript ${ }^{\mathrm{TM}}$ III, Invitrogen, USA) using random primers (Promega, USA) and Platinum $^{\circledR}$ SYBR $^{\circledR}$ Green qRT-PCR SuperMix-UDG (Invitrogen, Carlsbad, CA, USA) and analyzed on an ABI PRISM 7900 HT (PE Applied Biosystems, Foster

Table 1 Primer sequences used in quantitative qRT-PCR analyses

\begin{tabular}{|c|c|c|c|}
\hline Gene name & Gene symbol & $\begin{array}{c}\text { Sense primer } \\
\left(5^{\prime}-3^{\prime}\right)\end{array}$ & $\begin{array}{c}\text { Antisense primer } \\
\left(5^{\prime}-3^{\prime}\right)\end{array}$ \\
\hline 3-hydroxy-3-methylglutaryl-Coenzyme A reductase & $\mathrm{Hmgcr}^{1}$ & cttgtggaatgccttgtgattg & agccgaagcagcacatgat \\
\hline Low density lipoprotein receptor & $L d l r^{r}$ & aggctgtgggctccatagg & tgcggtccagggtcatct \\
\hline Insulin induced gene 1 & $\ln \operatorname{sig} 1^{2}$ & tcacagtgactgagcttcagca & tcatcttcatcacacccaggac \\
\hline Peroxisome proliferator activated receptor $\alpha$ & Ppara $^{3}$ & cctcttcccaaagctccttca & cgtcggactcggtcttcttg \\
\hline Deiodinase iodothyronine type $\|$ & $\operatorname{Dio} 2^{4}$ & \multicolumn{2}{|c|}{ Mm00515664_m1 } \\
\hline Interleukin-6 & $116^{4}$ & \multicolumn{2}{|c|}{ Mm00446190_m1 } \\
\hline Glucose- 6 phosphatase & $G 6 P C^{4}$ & \multicolumn{2}{|c|}{ Mm00839363_m1 } \\
\hline $18 \mathrm{~S}$ ribosomal RNA & $18 S^{5}$ & cgccgctagaggtgaaattc & ttggcaaatgctttcgctc \\
\hline$\beta$-actin & Actb & ccgtgaaaagatgacccagatc & cacagcctggatggctacgt \\
\hline
\end{tabular}

${ }^{1}$ Reference [41]; ${ }^{2}$ Reference [42]; ${ }^{3}$ Reference [43] ; ${ }^{4}$ Assay-on-demand (Applied Biosystems) ${ }^{5}$ Reference [44]. 
Table 2 Differential gene expression in skeletal muscle between the Lean and Fat mice

\begin{tabular}{lccc}
\hline $\begin{array}{l}\text { SKELETAL MUSCLE } \\
\text { (gene name) }\end{array}$ & $\begin{array}{c}\text { Gene } \\
\text { symbol }\end{array}$ & $\begin{array}{c}\log _{2} \text { ratio } \\
\text { (qRT-PCR) }\end{array}$ & L:F** $^{* *}$ \\
\hline Interleukin-6 & 116 & $0.71^{*}$ & $\uparrow$ \\
Deiodinase iodothyronine type II & Dio2 & $-0.5^{*}$ & $\downarrow$ \\
\hline
\end{tabular}

*P $<0.05$.

**Up arrow indicates increased mRNA expression in the Lean (L) line and down arrow decreased expression in the Lean line compared to the Fat $(F)$ line.

City, CA, USA) according to the manufacturer's protocols. Internal controls were $18 \mathrm{~S} r R N A$ for liver and $A c t b$ (beta-actin) for muscle. Relative transcript levels were statistically analyzed using the comparative $\mathrm{Ct}$ (cycle threshold) and $-\Delta \Delta \mathrm{Ct}$ values [23]. Single-factor

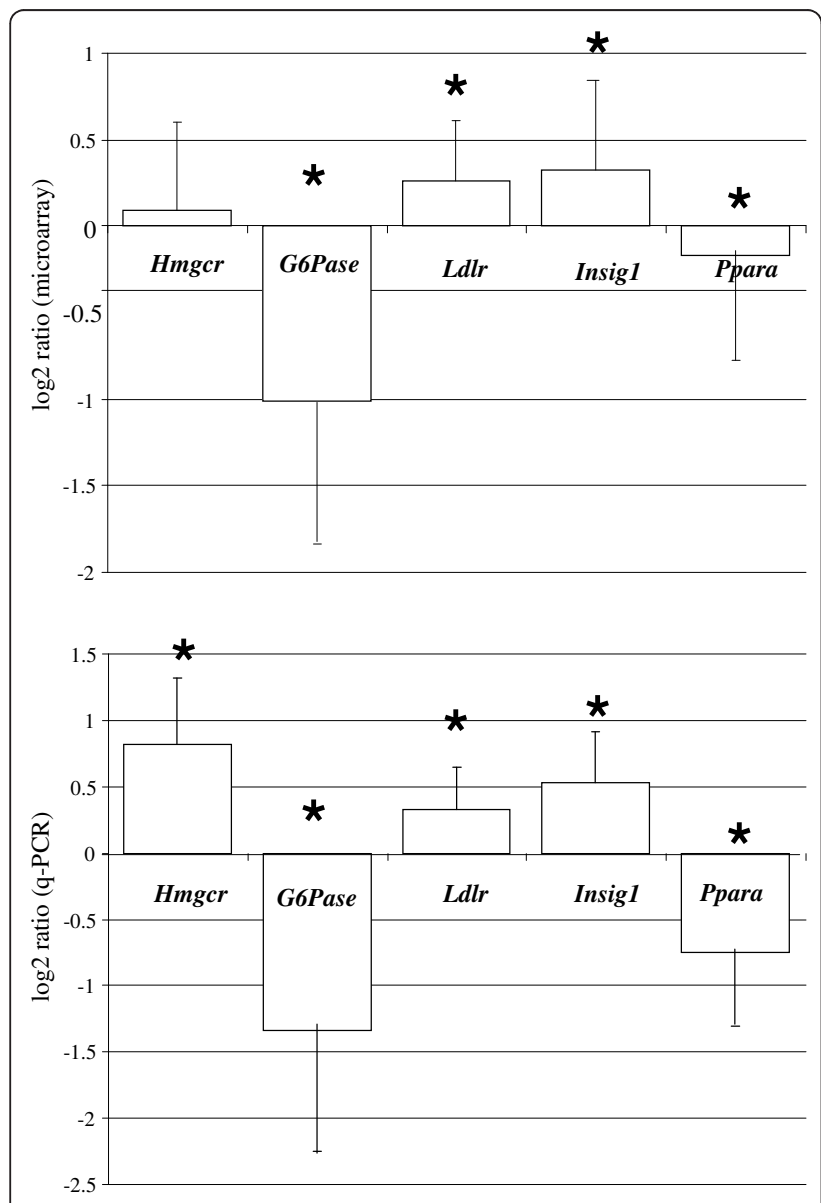

Figure 1 Comparison of randomly selected genes in the microarray experiment and by qRT-PCR method. Five genes shown to be differentially expressed by the microarray analysis (upper panel) were randomly selected for validation by qRT-PCR (lower panel). All five genes also showed statistical significant differences $\left({ }^{*} P<0.05\right)$ between the Lean and Fat line by qRT-PCR as well as the same direction of gene-expression differences supporting the credibility of microarray experiment. Average $\log _{2}$ ratios +/- standard errors are displayed. analysis of variance and a probability of type I error $\alpha=0.05$ was used to determine statistical significance in SPSS 14.0 (SPSS Inc., Chicago, Illinois, USA). Primer sequences are provided in Table 1.

\section{Results}

Lean mice exhibit a liver transcriptome profile suggesting improved cholesterol homeostasis, bile acid, glucose and lipoprotein metabolism

Analysis of Steroltalk v2 microarray data identified 38 differentially expressed genes $(\mathrm{P}<0.05)$ that were further separated into metabolic pathway groups such as cholesterol biosynthesis pathway, bile and glucose metabolism, drug metabolism and others (Table 3). Geneexpression differences as well as their statistical significances were confirmed by qRT-PCR (Figure 1). Expression of several genes involved in the cholesterol biosynthesis pathway was found to be elevated in Lean mice (Table 3), suggesting that liver cholesterol production might also be elevated compared to the Fat mice. In addition, the Insig1 gene, a potent regulator of cholesterol biosynthesis transcription factors and a modulator of HMG-CoA reductase protein ubiquitination, was also expressed at a higher level in Lean mice (Table 3).

Transporter genes involved in the uptake of plasma bile acids into hepatocytes such as Slco1a1, Slco1a4, Slco1b2, Slc10a1, and the cholesterol efflux transporter $A b c g 5$ were down-regulated in Lean mice. However, $A b c b 11$, which controls bile acid excretion, was up-regulated. Cyp 8 b1, a gene encoding a bile acid synthesis enzyme, was also expressed at a higher level in Lean mice (Table 3).

Gluconeogenesis genes G6Pc (glucose-6-phosphatase, catalytic) and $P d h b$ (pyruvate dehydrogenase beta) were markedly down-regulated in Lean mice supporting previously identified predisposition of Lean line to normoglycemia and resistance to diabetes development (Table 3).

The Ldlr, Lip1 and Adipor2 receptors were up-regulated in livers of Lean mice suggesting that livers of Lean mice have an increased ability to capture atherogenic LDL-cholesterol as well as to respond to the white adipose tissue derived hormone adiponectin. In contrast, the transcription factor Ppara, involved in triglyceride homeostasis, was down-regulated in Lean mice (Table 3).

Many of the cytochromes P450 functioning in drug and steroid hormone metabolism were down-regulated (Cyp2a5, Cyp2b10, Cyp2b9 and Cyp3a41) although two (Cyp2c69 and Cyp1a2) were up-regulated (Table 3). However, it is important to note that the cDNA probes used on the microarrays do not enable efficient differentiation between the aforementioned CYP members, which share a relatively high sequence homology. For example, probes for Cyp $2 b 10$ and Cyp $2 b 9$ cross-react 
Table 3 Differential expression between the Lean and Fat mice in liver genes

\begin{tabular}{|c|c|c|c|c|}
\hline Gene name & Gene Symbol & GeneBank Code & $\log _{2}$ ratio* $^{*}$ & $\mathrm{~L}: \mathrm{F}^{* *}$ \\
\hline \multicolumn{5}{|l|}{ Cholesterol metabolism } \\
\hline Mevalonate kinase & Mvk & NM_023556 & 0.11 & $\uparrow$ \\
\hline NAD(P) dependent steroid dehydrogenase-like & Nsdhl & BC019945 & 0.15 & $\uparrow$ \\
\hline Sterol-C5-desaturase (fungal ERG3, delta-5-desaturase) homolog (S. cerevisae) & $\operatorname{sc} 5 d$ & BC024132 & 0.27 & $\uparrow$ \\
\hline Mevalonate (diphospho) decarboxylase & Mvd & NM_138656 & 0.29 & $\uparrow$ \\
\hline Lanosterol synthase & Lss & NM_146006 & 0.33 & $\uparrow$ \\
\hline Farnesyl diphosphate synthetase & Fdps & NM_134469 & 0.41 & $\uparrow$ \\
\hline Farnesyl diphosphate farnesyl transferase 1 & Fdft 1 & NM_010191 & 0.44 & $\uparrow$ \\
\hline Acyl-CoA synthetase short-chain family member 2 & Acss2 & NM_019811 & 0.36 & $\uparrow$ \\
\hline \multicolumn{5}{|l|}{ Bile acid and glucose metabolism } \\
\hline Glucose-6-phosphatase, catalytic & G6PC & NM_008061 & -1.01 & $\downarrow$ \\
\hline Pyruvate dehydrogenase (lipoamide) beta & Pdhb & NM_024221 & -0.28 & $\downarrow$ \\
\hline Solute carrier organic anion transporter family, member 1a4 & Slcola4 & NM_030687 & -0.71 & \\
\hline Solute carrier organic anion transporter family, member 1a1 & Slcolar & AY195868 & -0.49 & $\downarrow$ \\
\hline Solute carrier organic anion transporter family, member $1 \mathrm{~b} 2$ & Slcolb2 & NM_020495 & -0.36 & $\downarrow$ \\
\hline Solute carrier family 10 (sodium/bile acid cotransporter family), member 1 & Slcioar & BC094023 & -0.13 & $\downarrow$ \\
\hline ATP-binding cassette, sub-family G (WHITE), member 5 & Abcg5 & NM_031884 & -0.15 & \\
\hline ATP-binding cassette, sub-family B (MDR/TAP), member 11 & Abcb11 & NM_021022 & 0.65 & $\uparrow$ \\
\hline Cytochrome P450, family 8, subfamily b, polypeptide 1 & Cyp8b1 & NM_010012 & 0.52 & $\uparrow$ \\
\hline \multicolumn{5}{|l|}{ Drug metabolism } \\
\hline Cytochrome P450, family 2, subfamily a, polypeptide 5 & Cyp2a5 & BC011233 & -0.52 & \\
\hline Cytochrome P450, family 2, subfamily b, polypeptide 10 & Cyp2b10 & AK028103 & -0.44 & $\downarrow$ \\
\hline Cytochrome P450, family 2, subfamily b, polypeptide 9 & Cyp2b9 & NM_010000 & -0.38 & $\downarrow$ \\
\hline Cytochrome P450, family 2, subfamily b, polypeptide 9 & Cyp2b9 & NM_007813 & -0.38 & $v_{V}$ \\
\hline Cytochrome P450, family 3, subfamily a, polypeptide $41 \mathrm{~A}$ & Cyp3a41a & NM_017396 & -0.37 & $\downarrow$ \\
\hline Cytochrome P450, family 2, subfamily c, polypeptide 69 & Cyp2c69 & NM_010004 & 0.34 & $\uparrow$ \\
\hline Cytochrome P450, family 1 , subfamily a, polypeptide 2 & Cypla2 & NM_009993 & 0.41 & $\uparrow$ \\
\hline \multicolumn{5}{|l|}{ Others } \\
\hline Complement component 9 & C9 & BC011137 & -1.37 & $\downarrow$ \\
\hline Serum amyloid P-component & Apcs & BC061125 & 0.46 & $\uparrow$ \\
\hline Complement component 4B (Childo blood group) & $C 4 b$ & BC067409 & 1.55 & $\uparrow$ \\
\hline C-reactive protein, pentraxin-related & Crp & NM_007768 & 0.25 & $\uparrow$ \\
\hline Orosomucoid 1 & Orm1 & BC012725 & -0.34 & $\downarrow$ \\
\hline Hemopexin & Hpxn & BC019901 & 0.12 & $\uparrow$ \\
\hline Nuclear receptor subfamily 5 , group A, member 2 (LRH-1) & $\mathrm{Nr} 5 \mathrm{a} 2$ & NM_030676 & 0.16 & $\uparrow$ \\
\hline Acetyl-Coenzyme A acetyltransferase 2 & Acat2 & NM_009338 & 0.22 & $\uparrow$ \\
\hline Apolipoprotein A-II & Apoa2 & BC031786 & 0.23 & $\uparrow$ \\
\hline Lysosomal acid lipase A & Lip1 & NM_021460 & 0.23 & $\uparrow$ \\
\hline Low density lipoprotein receptor & Ldlr & BC019207 & 0.27 & $\uparrow$ \\
\hline Insulin induced gene 1 & Insig1 & NM_153526 & 0.33 & $\uparrow$ \\
\hline Nitric oxide synthase 1, neuronal & Nos1 & BC066101 & 0.34 & $\uparrow$ \\
\hline Adiponectin receptor 2 & Adipor2 & NM_197985 & 0.35 & $\uparrow$ \\
\hline
\end{tabular}

*Data represent log2 ratios of Lean (L) versus Fat (F) line $(p<0.05)$.

**Up arrow indicates increased mRNA expression of particular gene in the Lean (L) line and down arrow decreased expression in the Lean line.

with other $C y p 2 b$ family members, and the same holds true for the Cyp2c, Cyp $3 a$ and Cyp $2 a$ probes. Therefore, to determine exactly which gene from the CYP2,3 families is differentially expressed in our lines, future experiment based on allele-specific hybridization probes or gene specific RT-PCR assays should be conducted.
Lean mice display an improved profile of adiposity, plasma lipoproteins, plasma bile acids and liver triglycerides parameters

Food intake analysis demonstrated statistically nonsignificant differences between Lean and Fat mice (data not shown) supporting previous findings that 
Lean and Fat mice, despite the large phenotypic difference, consume equal amounts of food [24]. Lean line males compared to the Fat males exhibited significantly lower body fatness at 14-weeks of age across all dissected fat depots (Table 4). The relative weight (normalized to body weight) of abdominal, femoral, epididymal and mesenterial fat of Lean mice was approximately 5.4, 4.0, 7.0 and 3.5 fold lower compared to Fat line males, respectively. On the contrary, the relative heart weight was 1.34 fold higher in Lean versus Fat mice.

Plasma was individually analyzed in Lean and Fat males for total plasma cholesterol (Figure 2A) and HDL/total plasma cholesterol ratio (Figure 2B). Non-HDL-cholesterol was calculated by subtracting total plasma cholesterol from HDL-cholesterol and the ratio of non-HDL-cholesterol/total plasma cholesterol was subsequently calculated (Figure 2C). The beneficial
Table 4 Weights of fat depots and heart normalized to body weight in Lean and Fat mice

\begin{tabular}{|c|c|c|c|}
\hline Parameter & Lean mice* & Fat mice* & No. of individuals \\
\hline $\begin{array}{l}\text { BODY WEIGHT at } \\
14 \text { weeks } \\
\text { (g) }\end{array}$ & $28.17 \pm 1.15$ & $41.76 \pm 3.22^{*}$ & $L=23 ; F=24$ \\
\hline ABDOMINAL FAT & & & \\
\hline $\begin{array}{l}\text { ratio (mg/g body wt) } \\
\text { FEMORAL FAT }\end{array}$ & $1.86 \pm 069$ & $9.97 \pm 1.08^{\mathrm{a}}$ & $L=43 ; F=42$ \\
\hline $\begin{array}{c}\text { ratio (mg/g body wt) } \\
\text { EPIDIDYMAL FAT }\end{array}$ & $4.43 \pm 1.2$ & $17.67 \pm 2.32^{a}$ & $L=43 ; F=42$ \\
\hline $\begin{array}{c}\text { ratio (mg/g body wt) } \\
\text { MESENTERIAL FAT }\end{array}$ & $3.34 \pm 0.85$ & $23.24 \pm 3.12^{\mathrm{a}}$ & $L=43 ; F=42$ \\
\hline $\begin{array}{c}\text { ratio (mg/g body wt) } \\
\text { HEART }\end{array}$ & $6.43 \pm 1.26$ & $22.33 \pm 3.87^{\mathrm{a}}$ & $L=18 ; F=15$ \\
\hline ratio (mg/g body wt) & $6.13 \pm 0.87$ & $4.58 \pm 0.69^{\mathrm{a}}$ & $L=43 ; F=42$ \\
\hline
\end{tabular}

*Results are expressed as means \pm S.D. Except for mesenterial fat and heart, means were calculated based on weighed left-sided fat depot (L; Lean mice, F; Fat mice, ${ }^{\text {a }} P<0.001$.
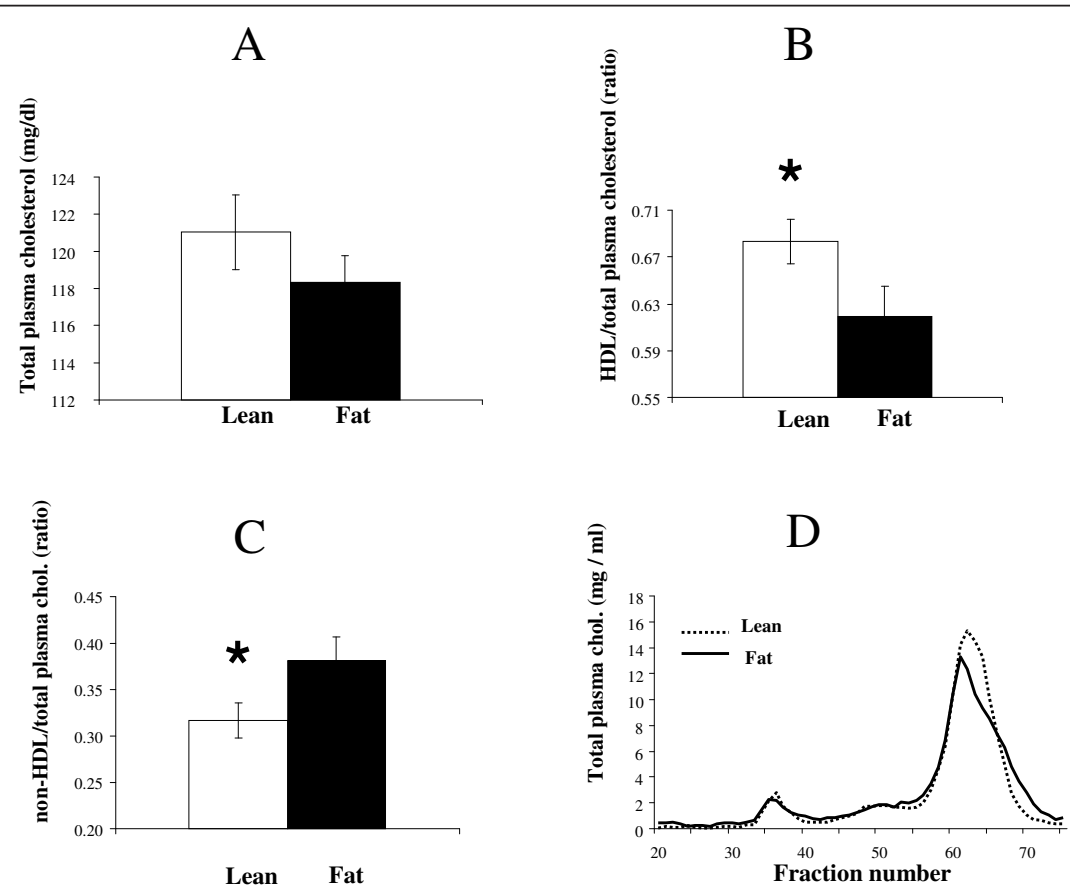

Figure 2 Detailed plasma cholesterol analyses in the Lean and Fat mice. A. Total plasma cholesterol concentrations. Although cholesterol biosynthesis genes showed increased expression in the liver of Lean mice (Table 3), total blood plasma cholesterol concentration was not significantly different to the Fat mice. Average concentrations of total plasma cholesterol (mg/dl) standard errors are displayed. B. Ratio of HDLcholesterol to total plasma cholesterol. Beneficial HDL-cholesterol is significantly $\left.{ }^{*} P<0.05\right)$ increased in Lean mice. Average ratios and standard errors are displayed. C. Ratio of nonHDL-cholesterol to total plasma cholesterol. nonHDL-cholesterol was a derived parameter obtained by subtracting total plasma cholesterol from HDL-cholesterol. As HDL-cholesterol is markedly elevated in Lean mice (Figure 2B), consequently the Lean mice have significantly ( ${ }^{*} P<0.05$ ) lower ratio of atherogenic nonHDL-cholesterol to total plasma cholesterol. Average ratios and standard errors are displayed. D. FPLC analysis of total plasma lipoproteins. Cholesterol distribution among lipoproteins was obtained by separation of the major lipoprotein classes (VLDL, IDL+LDL, and HDL) by fast protein liquid chromatography (FPLC). This analysis determined in the Lean line a higher peak in the HDL fraction as well as a shift of HDL particles towards increased fraction elution number, suggesting that both the size and concentration of plasma HDL lipoproteins are elevated in Lean mice. 
HDL-cholesterol was markedly elevated in Lean mice (Figure 2B) and consequently the Lean mice showed significantly lower ratios of atherogenic non-HDL-cholesterol versus total plasma cholesterol (Figure 2C). However, despite the higher expression level of genes involved in cholesterol biosynthesis in the Lean mice (Table 3), total plasma cholesterol concentration was not significantly different between the lines (Figure 2A). A follow up FPLC plasma analysis determined that the Lean line had a higher peak in the HDL fraction as well as a shift of the HDL curve suggesting that both the size and concentration of plasma HDL lipoproteins were elevated in Lean mice (Figure 2D).

Significantly higher concentrations of total plasma bile acids were found in Lean mice (Figure 3) as well as lower hepatic triglyceride levels (Figure 4A). However, total liver cholesterol analysis resulted in statistically non-significant differences (Figure 4B) suggesting that this unlikely presents a driving effect.

\section{Lean mice show increased skeletal muscle oxidative capacity}

Analysis of $m$. gastrocnemius SDH-activity determined by histochemical reaction identified marked differences in oxidative capacity of skeletal muscle fibres from Fat versus Lean mice. Significantly higher levels of SDH activity were detected in muscle fibres of Lean mice (Figure 5, P $<0.001$ ). Skeletal muscle interleukin 6 (Il6) mRNA level, a potent indicator of muscle physical activity, was examined using qRT-PCR. Lean mice muscle tissue displayed markedly elevated $I l 6$ gene expression level (Table 2). Furthermore, a significant down- regulation of iodothyronine, type II deiodinase (Dio2) in $m$. gastrocnemius and $m$. quadriceps of Lean mice (Table 2) was determined.

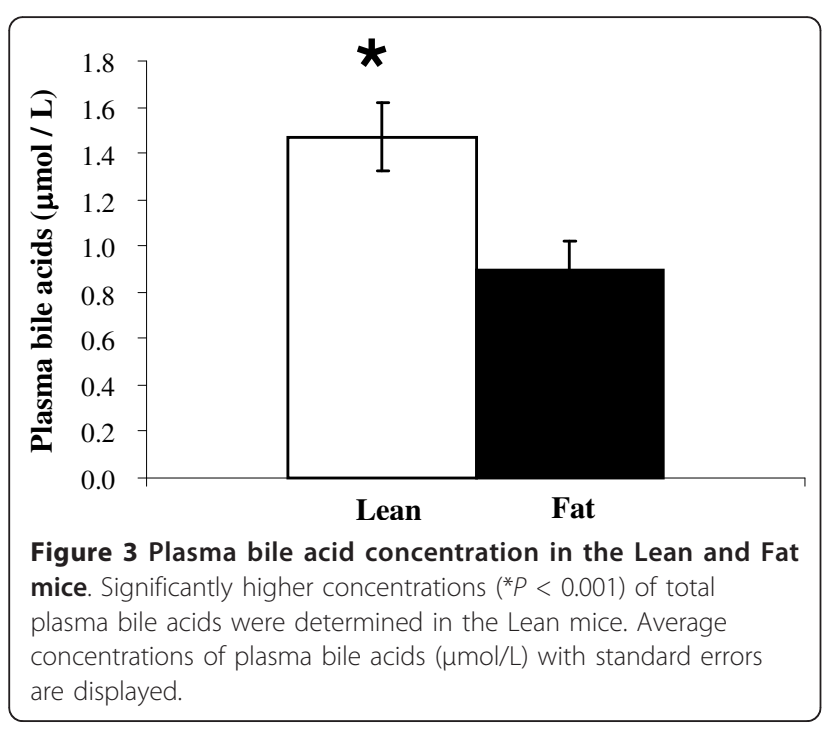

\section{Discussion}

The main objective of the present study was to identify candidate genes and molecular mechanisms responsible for obesity-resistance in the Lean line. Additionally, we aimed to investigate metabolic parameters of skeletal muscle and liver tissue and how they relate to blood lipoproteins, triglycerides, bile acids and liver transcriptome. Phenotypic characterization and search of differentially expressed genes was also carried out to identify novel positional candidate genes that map to the regions of quantitative trait loci determined in previous crosses between the Fat and Lean mice.

\section{Non-atherogenic lipoprotein profile in Lean mice}

Plasma and liver cholesterol concentrations are, under balanced diet conditions, determined by the affluence from hepatic de-novo cholesterol biosynthesis. Additionally, excretion rate of cholesterol and bile acids also contribute to inter-individual variation of total plasma and liver cholesterol.

In the current experiment a large number of liver cholesterol biosynthesis genes were upregulated in the Lean line, but this was not reflected in an elevation of total liver and plasma cholesterol. This inconsistency could be explained in two ways: 1) either upregulation of cholesterol biosynthesis genes was not sufficient to increase the metabolic flow in cholesterol biosynthesis pathway that would result in an increased level of de novo hepatic cholesterol; 2) or that novel and excess hepatic cholesterol was excreted from the liver or metabolized to bile acids. Since we have not measured the metabolic rate of hepatic cholesterol biosynthesis or determined protein levels, we can not confirm yet that the increase in mRNA expression resulted in increased metabolic rate of cholesterol biosynthesis. Regulation of the cholesterol synthesis genes is under control of the SREBP signalling pathway [25] and upregulation observed in the Lean line is probably a result of activation of the SREBP transcription factor cascade. This is supported by our finding that Insig-1 and $L d l r$, both being SREBP target genes, were up-regulated in the Lean mice. The hypothesis that cholesterol in the Lean mice would be excreted from the liver or metabolized to bile acids at an enhanced rate has limited supportive results. Higher efflux of cholesterol from the liver to bile is not likely since one of the genes involved in this process, Abcg5, is downregulated in the Lean mice. Increase in bile acid synthesis is plausible, since $C y p 8 b 1$ was up-regulated. However; Cyp $7 a 1$, a rate-limiting enzyme of bile acid synthesis, was not changed (data from RT-PCR not shown). Nevertheless, Cyp $7 a 1$ gene exhibits large interindividual differences in the level of expression and this hinders statistical significance. Also, we found that the nuclear receptor subfamily 5 , group A, member 2 (Nr5a2, also known as LRH-1), a regulator of 

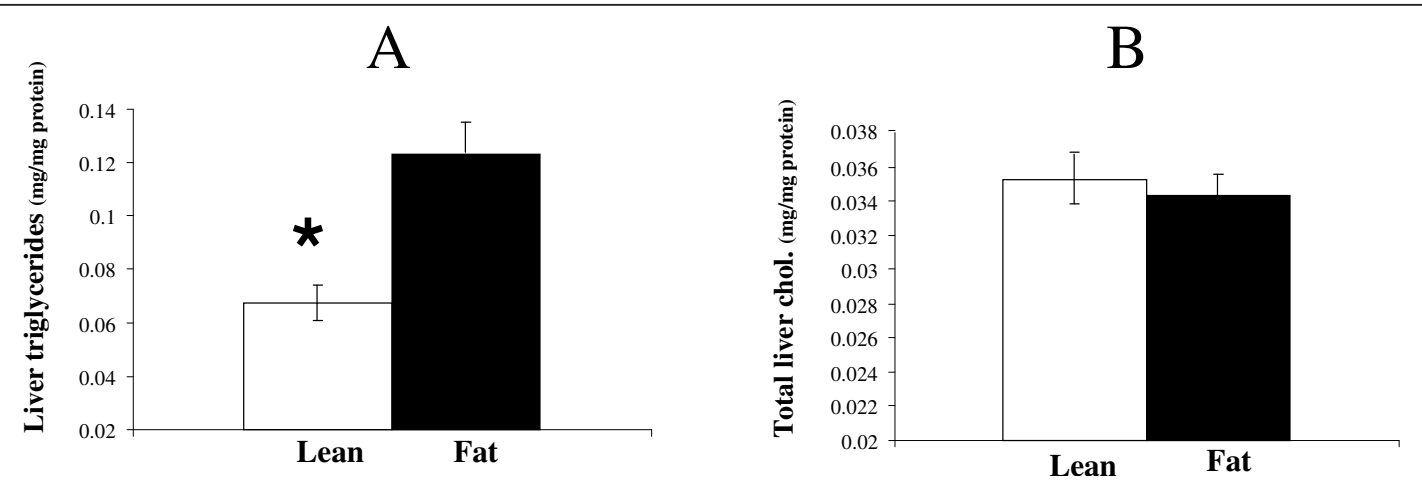

Figure 4 Triglyceride and total cholesterol content in the livers of Lean and Fat mice. A. Hepatic trygliceride levels. The liver of Lean mice contains significantly lower $\left({ }^{*} P<0.05\right)$ amounts of triglycerides. Average values for $\mathrm{mg}$ of triglycerides per $\mathrm{mg}$ of liver proteins with standard errors are displayed. B. Total hepatic cholesterol levels. Despite that cholesterol biosynthesis genes showed increased mRNA expression in the liver of Lean mice (Table 3), total liver cholesterol does not differ significantly from the Fat line. Average values for mg of total cholesterol per mg of liver proteins with standard errors are displayed.

bile acid synthesis genes, is upregulated in Lean mice. In order to fully explain why up-regulation of cholesterol pathway genes in the Lean line did not lead to increased levels of total liver and plasma cholesterol, future detailed studies should be focused on protein and metabolic level of cholesterol management in Lean and Fat lines.

Although we did not find statistically significant differences in total liver and plasma cholesterol between the lines, the Lean mice livers did contain significantly less triglycerides per mg of liver tissue than the Fat mice - Lean mice had only about $50 \%$ of Fat mice triglyceride content (Figure 4). $A b c b 11$, a gene overexpressed in the Lean line

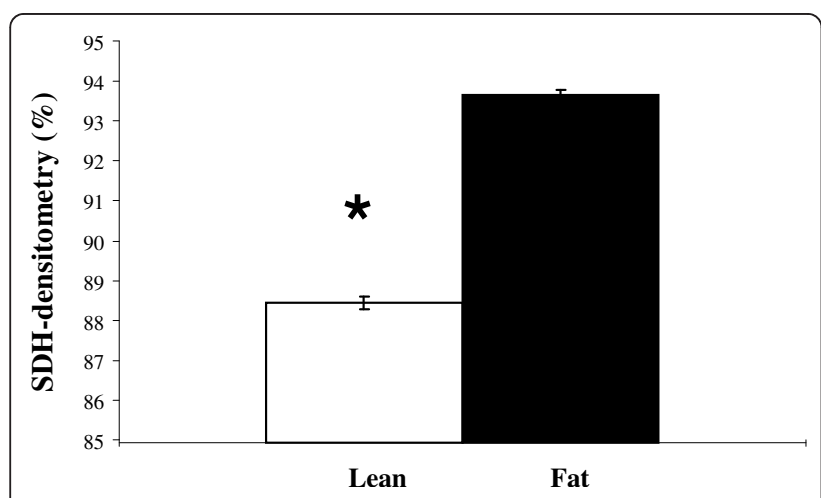

Figure 5 SDH-activity in $m$. gastrocnemius muscle tissue of the Lean and Fat mice. Striated muscle from regio cruris $(m$. gastrocnemius) was used to determine mitochondrial succinate dehydrogenase (SDH) activity levels. 12 of each Lean and Fat line mice were analysed and 150 muscle fibers per mouse were measured for densitometry. This histochemical assay demonstrated marked ( ${ }^{*} P<0.001$ ) increased level of SDH activity in muscle fibers of Lean mice suggesting pronounced increase in oxidative metabolism in this line. \% densitometry with standard errors are displayed - lower transparency of muscle histological sections in the Lean mice resulted from higher SDH-enzyme activity which in turn gave lower \% densitometry readings in the Lean line. liver, is known to enhance the rate of triglyceride excretion from the liver [26,27]. Also, in a transgenic $A b c b 11$ overexpression model [28], increased liver $A b c b 11$ expression showed ameliorating effects on hepatic steatosis by increasing excretion of biliary phospholipids and cholesterol. It is possible therefore that upregulation of $A b c b 11$ in the Lean mice might account for their diminished content of liver triglycerides. $A b c b 11$ is also a strong positional candidate because it maps within a QTL Fob1 on chromosome 2, detected in our previous cross between Lean and Fat mice [17]. Also supportive is a study in humans detecting 30 mutations in the $A B C B 11$ gene that were associated with different clinical phenotypes and triglyceride measurements [29]. Therefore, Abcb11 could be one of the potential causal allelic variants between the Lean and Fat mice, which can aid in explaining a part of the phenotype divergence between the strains. Elevated HDL and upregulation of some bile acids synthesis and transport genes suggests enhanced reverse cholesterol transport in the Lean line. As the cholesterol excretion rate is higher, Lean line compensates this by upregulating of endogenous cholesterol biosynthesis as demonstrated by the array experiment.

We established that Lean mice have significantly elevated plasma HDL. Interestingly, results from a previous study on humans imply that more exercise leads to increased total HDL and the average size of HDL particles [30]. FPLC analysis of plasma revealed similar observations - Lean mice have higher HDL plasma concentrations and also tend to have HDL particles of increased size. Interpretation for improved HDL profile in Lean mice can be further substantiated by genetic studies on HDL metabolism, where several QTLs were demonstrated to determine plasma HDL concentration, some also mapping to QTL regions detected in Fat and Lean mice $[17,31]$. It is therefore possible that some of the obesity QTLs identified in our previous genetic 
studies have pleiotropic effects affecting both body fat \% and HDL levels in our polygenic animal model [17-19]. The explanation for the observed higher concentration of HDL and lower concentration of LDL-cholesterol in Lean mice may therefore lie in allelic differences in some of the HDL QTLs between the Fat and Lean mice.

\section{Plasma bile acids and changes in skeletal muscle metabolism}

The bile acid pool in plasma is maintained by the enterohepatic recirculation. The uptake of bile acids that return to the liver after intestinal absorption is mainly mediated by a family of SLCO transporters (Slco1a1, Slco1a4, Slco10a1) [27] whose expression can be modulated by plasma bile acids and cytokines. In our Lean mice down-regulation of genes encoding the aforementioned hepatic bile acid transporters along with significantly elevated concentrations of plasma bile acids was detected. Other studies have also demonstrated that bile acids can down-regulate expression of Slco genes and up-regulate $A b c b 11$ [32], which is in agreement with our results obtained in the Lean line. Additional support comes from a study identifying significant dose-dependent mRNA suppression of Slco1a1 (Oatp1) and Slco1a4 (Oatp2) in mice treated with cytokine IL6 [33]. Detailed in-vitro and in-vivo studies defined IL6 as by far the most pronounced transcriptional regulator of, for example, Slco1a1, Slco1a4 and Abcb11 [33,34]. IL6, also termed as »exercise factor «, is highly expressed in contracting (exercising) skeletal muscle and is the most highly secreted systemic muscle cytokine during exercise [35]. The expression level of Il6 mRNA serves as a predictor of plasma IL6 protein [36]. In line with these reports, our analysis demonstrates high levels of expression of skeletal muscle Il6 mRNA in Lean mice. This increased $I l 6$ expression in the Lean line could be a consequence of increased physical activity of Lean mice reported in our previous experiments [37]. Thus, relatively high IL6 plasma levels in Lean mice might suppress the expression of the Slco family of genes subsequently leading to the increase in plasma bile acids. However, future studies are needed to confirm that the muscle-derived cytokine IL6 in the Lean mice is directly responsible for down-regulation of Slco genes and increase in plasma bile acids.

Because Lean mice contain elevated levels of plasma bile acids we sought to interpret other potential metabolic actions involving bile acids. Elevated levels of plasma bile acids were found to lead to endocrine actions with consequent impact on whole body energy homeostasis [38]. This effect is elicited through the increased mRNA expression of type II iodothyronine deiodinase (Dio2) which was exemplified in the mouse brown adipose tissue and in human skeletal muscle myoblasts [38].
Dio2 expression was elevated in our Lean mice and also maps close to the Fob2 QTL, previously identified as one of four loci significantly contributing to the divergent body fat $\%$ in our polygenic mouse model [17]. In humans, polymorphisms at the Dio2 locus determines susceptibility to hypertension and diabetes $[39,40]$. Given the expression difference in the present study and its location to previously mapped QTL in the same mice, it is plausible that the Dio 2 allelic variant also exists in this animal model, and may consequently determine differential expression and downstream consequences on phenotype in the Lean line.

The overall results of our current study are in line with our previous energy budget results which suggested that Lean mice expend more energy on physical activity [11] and our follow-up study demonstrating higher running wheel activity as well as higher exercise-independent posture allocation to more energy demanding positions (Non-Exercise Activity Thermogenesis; NEAT-standing, fidgeting, etc.) in Lean mice [37]. Increased oxidative capacity of muscle determined in the current study and resulting increased levels of Il6 expression may therefore indeed stem from higher physical and NEAT activities leading to downstream favourable effects on the liver transcriptome, cholesterol, bile acid, glucose and lipoprotein homeostasis.

However, the identified differences in liver transcriptome and other parameters and their associations with muscle metabolism and physical activity can explain only a part of a large phenotypic divergence between the Lean and Fat line. In a polygenic model it is expected that many alleles at different loci will contribute cumulatively to obesity resistance in various components of energy intake and energy expenditure. Since we showed in this study that Lean mice have similar food intake to the Fat line, we can exclude hyperphagia or genetic differences in energy intake as a major component for the phenotypic divergence between the lines. However, we have already shown earlier that thermoregulation [11] may also be increased in the Lean line and potential differences in resting metabolic rate can not be excluded. Therefore, more comprehensive experiments monitoring behavioural traits in combination with metabolic changes to measure differences in fuel oxidation will be needed to provide a better basis for future quantitative evaluations of energy expenditure components and fuel utilisation. Our current study reveals several candidate genes as well as important target tissues on which to base new targeted hypotheses to be tested in the future.

\section{Conclusions}

Polygenic mouse models of leanness/obesity used in our study comprise complex obesity-resistant (Lean line) 
and obesity-susceptible (Fat line) gene networks. This study was designed to help identify candidate genes and reveal some metabolic mechanisms responsible for the phenotypic difference between these lines. Results of liver transcriptome analysis along with markers of oxidative capacity (SDH enzyme activity, Il6 expression) in skeletal muscle suggests of multiple tissue metabolic interactions in the obesity resistant Lean line. Candidate genes, particularly those mapping to previously detected QTL regions (Abcb11 and Dio2) between Lean and Fat mice, and new phenotypic parameters obtained in this study will give us leads to identify novel genes underlying this complex network. The results of the present study combined with previous findings help to elucidate, at least in part, a molecular basis for the contrasting phenotype between the Lean and Fat mice and substantiate a hypothesis concerning metabolic interactions between the physical activity, skeletal muscle and liver tissue. We demonstrate that the Lean mouse line is a potentially good model for identifying novel genes and mechanisms in obesity resistance.

\section{Additional material}

Additional file 1: List of genes contained on the custom Steroltalk v2 microarray prepared for this study.

\begin{abstract}
Acknowledgements
We thank the Slovenian Research Agency for co-financing this work (core funding program P4-0220, project Syntol and Young Investigator grant). We are grateful to the Centre for functional genomics and biochips, Ljubljana, Slovenia http://cfgbc.mf.uni-lj.si/ for collaboration in the part of microarray analysis. We would also like to thank Zala Prevoršek, Nataša Debeljak, Nataša Toplak, Gorazd Tompa, Andrej Razpet, Ana Zanjkovič and Irena Oven for technical support and Emmanuelle Vallez for expert technical assistance and Jim McWhir for proofreading the manuscript. Finally, we are grateful to anonymous reviewers for meticulous review and constructive criticism that helped us to improve this manuscript.
\end{abstract}

\section{Author details}

'University of Ljubljana, Biotechnical Faculty, Department of Animal Science, Groblje 3, 1230 Domžale, Slovenia. ${ }^{2}$ University of Ljubljana, Institute of Biochemistry, Medical Faculty, Vrazov trg 2, 1000 Ljubljana, Slovenia. ${ }^{3}$ Institute of Anatomy, Histology and Embryology, Veterinary faculty, University of Ljubljana, Gerbičeva 60, 1000 Ljubljana, Slovenia. ${ }^{4}$ University Lille Nord de France, F-59000, Lille, France. ${ }^{5}$ Inserm, U1011, F-59000, Lille, France. ${ }^{6}$ UDSL, F-59000, Lille, France. ${ }^{7}$ Institut Pasteur de Lille, F-59019, Lille, France. ${ }^{8}$ National Institute of Chemistry, Hajdrihova 19, SI-1001 Ljubljana, Slovenia.

\section{Authors' contributions}

SH initiated, designed and co-ordinated the study, MS also participated in the design, performed animal experiments, RNA extraction, participated in the microarray hybridizations, GRT-PCR analyses, statistical analyses and drafted the manuscript; DR participated in the co-ordination of microarray studies and its experimental design, TR participated in the microarray and qRT-PCR experiments, PJ performed analysis of microarray data, CF and BS performed lipoprotein analyses and helped interpret phenotypic results, GF was involved in the experiment examining oxidative capacity of the muscle fibres. All authors contributed scientifically to the content of this study and have read and approved the final version of submitted manuscript.
Received: 22 August 2010 Accepted: 3 February 2011

Published: 3 February 2011

\section{References}

1. Batsis JA, Nieto-Martinez RE, Lopez-Jimenez F: Metabolic syndrome: From global epidemiology to individualized medicine. Clin Pharmacol Ther 2007, 82(5):509-524.

2. Weinsier RL, Hunter GR, Heini AF, Goran MI, Sell SM: The etiology of obesity: Relative contribution of metabolic factors, diet, and physical activity. Am J Med 1998, 105(2):145-150.

3. Neel JV: The "thrifty genotype" in 1998. Nutr Rev 1999, 57(5):S2-S9.

4. Butler AA, Cone RD: The melanocortin receptors: Lessons from knockout models. Neuropeptides 2002, 36(2-3):77-84.

5. Romao I, Roth J: Genetic and environmental interactions in obesity and type 2 diabetes. J Am Diet Assoc 2008, 108(4):S24-S28.

6. Ichihara S, Yamada Y: Genetic factors for human obesity. Cell Mol Life Sci 2008, 65(7-8):1086-1098.

7. Brockmann GA, Bevova MR: Using mouse models to dissect the genetics of obesity. Trends Genet 2002, 18(7):367-376

8. Speakman J, Hambly C, Mitchell S, Krol E: The contribution of animal models to the study of obesity. Laboratory animals 2008, 42(4):413-432.

9. Bunger L, Hill WG: Inbred lines of mice derived from long-term divergent selection on fat content and body weight. Mamm Genome 1999, 10(6):645-648.

10. Morton NM, Densmore V, Wamil M, Ramage L, Nichol K, Bunger L, Seckl JR, Kenyon CJ: A polygenic model of the metabolic syndrome with reduced circulating and intra-adipose glucocorticoid action. Diabetes 2005, 54(12):3371-3378.

11. Bunger L, Forsting J, McDonald KL, Horvat S, Duncan J, Hochscheid S, Baile CA, Hill WG, Speakman JR: Long-term divergent selection on body fatness in mice indicates a regulation system that is independent of leptin production and reception. Faseb J 2002, 16(13):85.

12. Simoncic M, Rezen T, Juvan P, Fievet C, Staels B, Rozman D, Horvat S: Transcriptome analysis revealed association of some $\mathrm{P} 450$ genes with obesity in a polygenic obese mouse model. Acta Chim Slov 2008, 55(1):101-110.

13. Tacer KF, Kuzman D, Seliskar M, Pompon D, Rozman D: TNF-alpha interferes with lipid homeostasis and activates acute and proatherogenic processes. Physiol Genomics 2007, 31(2):216-227.

14. Rezen $T$, Juvan P, Fon Tacer K, Kuzman D, Roth A, Pompon D, Aggerbeck LP, Meyer UA, Rozman D: The Sterolgene v0 cDNA microarray: a systemic approach to studies of cholesterol homeostasis and drug metabolism. BMC Genomics 2008, 9:76.

15. Conklin LS O-HM: Nutritional considerations in pediatric inflammatory bowel disease. Expert Review of Gastroenterology \& Hepatology 2010, 4(3):305-317.

16. Sharp GL, Hill WG, Robertson A: Effects of Selection on Growth, BodyComposition and Food-Intake in Mice .1. Responses in Selected Traits. Genet Res 1984, 43(1):75-92.

17. Horvat S, Bunger L, Falconer VM, Mackay P, Law A, Bulfield G, Keightley PD: Mapping of obesity QTLs in a cross between mouse lines divergently selected on fat content. Mamm Genome 2000, 11(1):2-7.

18. Stylianou IM, Christians JK, Keightley PD, Bunger L, Clinton M, Bulfield G, Horvat S: Genetic complexity of an obesity QTL (Fob3) revealed by detailed genetic mapping. Mamm Genome 2004, 15(6):472-481.

19. Prevorsek Z, Gorjanc G, Paigen B, Horvat S: Congenic and bioinformatics analyses resolved a major-effect Fob3b QTL on mouse Chr 15 into two closely linked loci. Mamm Genome 2010, 21(3-4):172-185.

20. Nachlas MM TK, De Souza E, Cheng CS, Seligman AM: Cytochemical demonstration of succinic dehydrogenase by the use of a new $p$ nitrophenyl substituted ditetrazole. J Histochem Cytochem 1957, 5:420-436.

21. Curk T, Demsar J, Xu QK, Leban G, Petrovic U, Bratko I, Shaulsky G, Zupan B: Microarray data mining with visual programming. Bioinformatics 2005, 21(3):396-398.

22. Brazma A, Hingamp P, Quackenbush J, Sherlock G, Spellman P, Stoeckert C, Aach J, Ansorge W, Ball CA, Causton HC, et al: Minimum information about a microarray experiment (MIAME) - toward standards for microarray data. Nature Genet 2001, 29(4):365-371.

23. Livak KJ, Schmittgen TD: Analysis of relative gene expression data using real-time quantitative PCR and the $2(T)$ (-Delta Delta C) method. Methods 2001, 25(4):402-408. 
24. Bunger L, Forsting J, McDonald KL, Horvat S, Duncan J, Hochscheid S, Baile CA, Hill WG, Speakman JR: Long-term divergent selection on fatness in mice indicates a regulation system independent of leptin production and reception. Faseb J 2003, 17(1):85-87.

25. Engelking LJ, Liang GS, Hammer RE, Takaishi K, Kuriyama H, Evers BM, Li WP, Horton JD, Goldstein JL, Brown MS: Schoenheimer effect explained - feedback regulation of cholesterol synthesis in mice mediated by Insig proteins. J Clin Invest 2005, 115(9):2489-2498.

26. Stieger B, Meier Y, Meier PJ: The bile salt export pump. Pflugers Arch 2007, 453(5):611-620.

27. Geier A, Wagner M, Dietrich CG, Trauner M: Principles of hepatic organic anion transporter regulation during cholestasis, inflammation and liver regeneration. Biochim Biophys Acta-Mol Cell Res 2007, 1773(3):283-308.

28. Figge A, Lammert F, Paigen B, Henkel A, Matern S, Korstanje R, Shneider BL, Chen F, Stoltenberg E, Spatz K, et al: Hepatic overexpression of murine Abcb11 increases hepatobiliary lipid secretion and reduces hepatic steatosis. J Biol Chem 2004, 279(4):2790-2799.

29. Pauli-Magnus C, Stieger B, Meier Y, Kullak-Ublick GA, Meier PJ: Enterohepatic transport of bile salts and genetics of cholestasis. J Hepatol 2005, 43(2):342-357.

30. Kraus WE, Houmard JA, Duscha BD, Knetzger KJ, Wharton MB, McCartney JS, Bales CW, Henes S, Samsa GP, Otvos JD, et al: Effects of the amount and intensity of exercise on plasma lipoproteins. N Engl J Med 2002, 347(19):1483-1492.

31. Wang XS, Paigen B: Genetics of variation in HDL cholesterol in humans and mice. CircRes 2005, 96(1):27-42.

32. Fickert P, Zollner G, Fuchsbichler A, Stumptner C, Pojer C, Zenz R, Lammert F, Stieger B, Meier PJ, Zatloukal K, et al: Effects of ursodeoxycholic and cholic acid feeding on hepatocellular transporter expression in mouse liver. Gastroenterology 2001, 121(1):170-183.

33. Hartmann G, Cheung AKY, Piquette-Miller M: Inflammatory cytokines, but not bile acids, regulate expression of murine hepatic anion transporters in endotoxemia. J Pharmacol Exp Ther 2002, 303(1):273-281.

34. Siewert E, Dietrich CG, Lammert F, Heinrich PC, Matern S, Gartung C, Geier A: Interleukin-6 regulates hepatic transporters during acute-phase response. Biochem Biophys Res Commun 2004, 322(1):232-238.

35. Pedersen BK, Akerstrom TCA: Role of myokines in exercise and metabolism. J Appl Physiol 2007, 103(3):1093-1098.

36. Febbraio MA, Pedersen BK: Muscle-derived interleukin-6: mechanisms for activation and possible biological roles. Faseb J 2002, 16(11):1335-1347.

37. Simoncic M, Horvat S, Stevenson PL, Bunger L, Holmes MC, Kenyon CJ, Speakman JR, Morton NM: Divergent physical activity and novel alternative responses to high fat feeding in polygenic fat and lean mice. Behav Genet 2008, 38(3):292-300.

38. Watanabe M, Houten SM, Mataki C, Christoffolete MA, Kim BW, Sato H, Messaddeq N, Harney JW, Ezaki O, Kodama T, et al: Bile acids induce energy expenditure by promoting intracellular thyroid hormone activation. Nature 2006, 439(7075):484-489.

39. Mentuccia D, Proietti-Pannunzi L, Tanner K, Bacci V, Pollin TI, Poehlman ET, Shuldiner AR, Celi FS: Association between a novel variant of the human type 2 deiodinase gene Thr92Ala and insulin resistance - Evidence of interaction with the Trp64Arg variant of the beta-3-adrenergic receptor. Diabetes 2002, 51(3):880-883.

40. Gumieniak O, Perlstein TS, Williams JS, Hopkins PN, Brown NJ, Raby BA, Williams GH: Ala92 type 2 deiodinase allele increases risk for the development of hypertension. Hypertension 2007, 49(3):461-466.

41. Yang J, Goldstein JL, Hammer RE, Moon YA, Brown MS, Horton JD: Decreased lipid synthesis in livers of mice with disrupted Site-1 protease gene. Proc Natl Acad Sci USA 2001, 98(24):13607-13612.

42. Yabe D, Komuro R, Liang GS, Goldstein JL, Brown MS: Liver-specific mRNA for Insig-2 down-regulated by insulin: Implications for fatty acid synthesis. Proc Natl Acad Sci USA 2003, 100(6):3155-3160.

43. Patel DD, Knight BL, Wiggins D, Humphreys SM, Gibbons GF: Disturbances in the normal regulation of SREBP-sensitive genes in PPAR alphadeficient mice. J Lipid Res 2001, 42(3):328-337.

44. Gutala RV, Reddy PH: The use of real-time PCR analysis in a gene expression study of Alzheimer's disease post-mortem brains. J Neurosci Methods 2004, 132(1):101-107. doi:10.1186/1471-2164-12-96

Cite this article as: Simončič et al:: Obesity resistant mechanisms in the Lean polygenic mouse model as indicated by liver transcriptome and expression of selected genes in skeletal muscle. BMC Genomics 2011 12:96.

\section{Submit your next manuscript to BioMed Central and take full advantage of:}

- Convenient online submission

- Thorough peer review

- No space constraints or color figure charges

- Immediate publication on acceptance

- Inclusion in PubMed, CAS, Scopus and Google Scholar

- Research which is freely available for redistribution

Submit your manuscript at www.biomedcentral.com/submit
Biomed Central 